Ramos-Álvarez, J.J.; Jiménez-Borrero, F.A.; Paredes-Hernández, V.; Gallardo, J.M.; RomeroMoraleda, B. (2021) High Intensity Efforts During Competition in Professional Football. Revista Internacional de Medicina y Ciencias de la Actividad Física y el Deporte vol. 21 (81) pp. 131-141 Http://cdeporte.rediris.es/revista/revista81/artperiodos1213.htm

DOI: https://doi.org/10.15366/rimcafd2021.81.009

\title{
ORIGINAL
}

\section{ESFUERZOS DE ALTA INTENSIDAD DURANTE LA COMPETICIÓN EN EL FÚTBOL PROFESIONAL}

\section{HIGH INTENSITY EFFORTS DURING COMPETITION IN PROFESSIONAL FOOTBALL}

\author{
Ramos-Álvarez, J.J.'; Jiménez-Borrero, F.A.'; Paredes-Hernández, V. ${ }^{3}$; \\ Gallardo, J.M. ${ }^{4}$ y Romero-Moraleda, B. ${ }^{5}$ \\ ${ }^{1}$ Doctor en Medicina y Cirugía. Especialista en Medicina de la Educación Física y el Deporte. \\ Departamento de Radiología, Rehabilitación y Fisioterapia. Universidad Complutense de \\ Madrid (España) jiramosa@ucm.es \\ 2 Especialista en Medicina de la Educación Física y el Deporte. Servicios Médicos del CF Rayo \\ Majadahonda. Servicios Médicos del At de Madrid, SAD (España) fabio jimenezb@hotmail.com \\ 3 Doctor en Ciencias de la Actividad Física y del Deporte. Facultad de Educación y Salud. \\ Universidad Camilo José Cela. Madrid (España). Servicios Médicos Real Madrid CF. \\ vparedes@ucjc.edu \\ 4 Doctora en Ciencias de la Actividad Física y del Deporte. Facultad de Educación y Salud. \\ Universidad Camilo José Cela. Madrid (España) jgperez@ucjc.edu \\ 5 Profesora Doctora Departamento de Educación Física, Deporte y Motricidad Humana. \\ Universidad Autónoma de Madrid (España) blanca.romero@uam.es
}

Código UNESCO/UNESCO Code: 3299 Medicina del Deporte/Sport Medicine. Clasificación del Consejo de Europa/ Council of Europe classification: 11 Medicina del Deporte/ Sport medicine.

Recibido 31 de enero de 2019 Received January 31, 2019

Aceptado 6 de junio de 2019 Accepted June 6, 2019

\section{RESUMEN}

El objetivo de este trabajo fue cuantificar el número y duración de los esfuerzos de muy alta intensidad y esprint (EMAVS) y el intervalo de recuperación entre ellos en 36 futbolistas profesionales de primera y de segunda división. Los jugadores fueron monitorizados a través de dispositivos GPS durante dos partidos oficiales.

La duración promedio de los EMAVS fue de 2,58 $\pm 0,44$ segundos con un intervalo medio entre los mismos de 107,07 $\pm 67,00 \mathrm{sg}$. La FC media durante los EMAVS se situó en 164,92 12,39 p.p.m., alcanzando el $85 \%$ de la FC máxima. No se 
encontraron diferencias significativas entre la duración de los EMAVS, los intervalos entre ellos y la FC alcanzada ( $p \leq 0,05$ en todos los casos), con gran variabilidad individual.

La utilización de la duración de los EMAVS, los tiempos de recuperación y la FC alcanzada durante los mismos, serían de gran utilidad para diseñar los entrenamientos interválicos en el fútbol.

PALABRAS CLAVE: entrenamiento interválico; sprint, fútbol, rendimiento, carrera de alta velocidad

\section{ABSTRACT}

The aim, of this work was to quantify the number and duration of the very highspeed running and sprinting events (VHSRS) and the recovery interval between them in 36 first and second division professional soccer players. The players were monitored through GPS devices during two official matches.

The average duration of the EMAVS was $2.58 \pm 0.44$ seconds with an average interval between them of $107.07 \pm 67.00 \mathrm{~s}$. The mean heart rate $(H R)$ during the EMAVS was $164.92 \pm 12.39$ p.p.m., reaching $85 \%$ of the maximum HR. No significant differences were found between the duration of the EMAVS, the intervals between them and the HR reached ( $\leq \leq 0.05$ in all cases), with great individual variability.

The use of the duration of the VHSRS, the recovery times and the HR reached during them, would be very useful to design the interval training in football.

KEY WORD: interval training; sprint, soccer, football, performance, high-speed running

\section{INTRODUCCIÓN}

El éxito en la competición depende en gran medida del rendimiento físico de los futbolistas. Existe una gran variedad de estudios para evaluar dicho rendimiento, pero actualmente se necesitan métodos más concretos para valorar sus capacidades físicas (Juárez-Toledo, Domínguez-García, Laguna-Camacho, Sotomayor-Serrano y Balbás-Lara, 2018). Además, hoy en día, el nivel y el número de competiciones en las que participan los equipos de fútbol es alto, muchos partidos en cortos espacios de tiempo. Estas variables condicionan unas demandas físicas elevadas, aspectos que condicionan el rendimiento (GarcíaConcepción, Peinado, Paredes-Hernández, \& Alvero-Cruz, (2015).

La capacidad de los futbolistas para repetir esfuerzos intermitentes de muy alta velocidad y esprint ( $\geq 19,8 \mathrm{~km} \mathrm{/} \mathrm{h})$ (EMAVS) (Bishop, Girard, \& MendezVillanueva, 2011; Rampinini et al., 2007), es una de las variables que puede contribuir a determinar su rendimiento, debido a que en estos períodos de alta 
intensidad es donde se realizan las acciones decisivas del partido (Bishop et al., 2011; Buchheit, Simpson, \& Mendez-Villanueva, 2013; Girard, MendezVillanueva, \& Bishop, 2011; Stolen, Chamari, Castagna, \& Wisloff, 2005; Wragg, Maxwell, \& Doust, 2000). Cuando los períodos de recuperación entre EMAVS duran más de $60 \mathrm{~s}$, la capacidad de repetir el esfuerzo requerido en la acción posterior no se ve afectado (Bishop \& Claudius, 2005; Duffield, King, \& Skein, 2009). Sin embargo, cuando la duración es menor de $60 \mathrm{~s}$, el rendimiento del jugador puede verse mermado, no alcanzado la intensidad que requiere esa acción de juego (Bishop, Edge, Davis, \& Goodman, 2004; Girard et al., 2011). Aunque estos eventos pueden representar solo el 0,5-3\% de la actividad de un futbolista durante un partido (Buchheit, Mendez-villanueva, Simpson, \& Bourdon, 2010; Stolen et al., 2005), la aleatorización en la repetición de los mismos puede, en algunos casos, dejar un tiempo insuficiente para la recuperación completa. La capacidad de repetirlos dependerá en gran medida, del metabolismo oxidativo del jugador y su capacidad de resintetizar los depósitos de fosfocreatina, amortiguación de $\mathrm{H}^{+}$y de factores neuromusculares como la excitabilidad muscular, la transmisión adecuada de los impulsos nerviosos y el reclutamiento de fibras musculares (Bishop et al., 2011; Gantois et al., 2017; Haseler, Hogan, \& Richardson, 1999; Rodriguez-Fernandez, Sanchez Sanchez, RodriguezMarroyo, Casamichana, \& Villa, 2017).

Algunos autores informan que los futbolistas profesionales tienen una mayor capacidad de realizar esfuerzos máximos que los futbolistas de categorías inferiores (Andersson, Randers, Heiner-Moller, Krustrup, \& Mohr, 2010; Impellizzeri et al., 2008), lo que podría ayudar a determinar la categoría en la que un jugador pudiera competir (Gabbett \& Mulvey, 2008). Por otro lado, también se ha informado que el entrenamiento interválico a la máxima intensidad mejora la capacidad de los jugadores de deportes de equipo (Taylor, Macpherson, Spears, \& Weston, 2015). Sin embargo, cualquier aplicación específica del entrenamiento interválico (IT) exige que se tenga en cuenta el tipo de deporte que se practica, la duración del período entre EMAVS y las características individuales de los propios deportistas (Viana et al., 2018).

Actualmente, el uso de las nuevas tecnologías en el fútbol ha permitido analizar exhaustivamente las demandas físicas y técnico-tácticas en competición. La utilización de dispositivos GPS con acelerómetro y banda de frecuencia cardiaca (FC) incorporados permite conocer variables de volumen e intensidad tanto en competición como en entrenamiento. Sin embargo, son muchos los estudios que describen estos parámetros promediados, sin conocer qué ocurre entre los periodos de alta intensidad(Bradley, Di Mascio, Peart, Olsen, \& Sheldon, 2010; Buchheit et al., 2013; Di Salvo, Gregson, Atkinson, Tordoff, \& Drust, 2009; Impellizzeri et al., 2008). Debido a esa falta de información que permitiría tener en cuenta la duración de los periodos de alta intensidad y la recuperación media entre los mismos para modelar el entrenamiento, el objetivo del presente trabajo fue registrar el número y la duración de los EMAVS competición en jugadores de fútbol de primera y segunda división española. Como objetivo secundario se comparó el número y duración de los EMAVS entre los jugadores de primera y segunda división y entre las diferentes posiciones tácticas. 


\section{MATERIAL Y MÉTODOS}

\section{Diseño del estudio}

Este trabajo se diseñó como un estudio de casos comparativo, transversal (nivel de evidencia III). Dada su naturaleza no invasiva y la importancia de los datos obtenidos con respecto al entrenamiento y competición de los jugadores, no se requirió la aprobación del comité de ética. Todos los datos se trataron de forma anónima, y los sujetos del estudio conocían solo sus propios resultados. Todos los sujetos proporcionaron su consentimiento informado para ser incluidos, de acuerdo con los principios de la Declaración de Helsinki con respecto a la investigación en sujetos humanos.

\section{Procedimiento}

Los sujetos de estudio fueron 36 futbolistas profesionales masculinos; 21 pertenecientes a dos equipos de la primera división española, y 15 a dos equipos de la segunda división española (edad 24,3 $\pm 2,9$ años, estatura 180,0 $\pm 0,07 \mathrm{~cm}$ y peso $75,13 \pm 7.59$ ). Todos se ofrecieron voluntariamente para participar. Los datos fueron recogidos durante dos partidos seleccionados al azar entre lo jugados por sus equipos. Se recogieron un total de 72 registros.

Todos los equipos involucrados tenían planes de entrenamiento similares: realizaban 5 sesiones de entrenamiento semanales más partido. El plan semanal consistía en: sesión de recuperación/compensatorio el día posterior a partido, descanso, 3 sesiones de adquisición táctica, más sesión de activación el día anterior a competir de nuevo. Además de la división en la que jugaron, los jugadores se agruparon según su posición táctica, es decir, defensas ( $n=8$ entre los jugadores de la primera división y 6 entre los jugadores de la segunda división), centrocampistas ( $n=9$ y 5 respectivamente) y delanteros ( $n=4$ y 4 respectivamente).

Durante los partidos seleccionados, los jugadores fueron monitorizados a través de un dispositivo GPS (GPSports EVO $10 \mathrm{~Hz}$. Camberra. Australia) con acelerómetro incorporado $(100 \mathrm{~Hz})$ que se colocaban por medio de un chaleco entre las escápulas que también registraba por medio de un sensor la FC. Se registraron los siguientes datos: el número de EMAVS realizados $a \geq 19,8 \mathrm{~km} / \mathrm{h}$, la duración de cada uno, el intervalo entre ellos y la FC alcanzada durante ellos. Para utilizar los datos de un jugador, tenía que haber jugado durante al menos 80 minutos. Todos los datos fueron recolectados en septiembre (el primer mes) de la temporada 2017-2018.

\section{Análisis estadístico}

Se utilizó la prueba de Kolmogorov-Smirnov para confirmar que las variables cuantitativas mostraron una distribución normal. Se realizó la ANOVA para detectar diferencias entre la división y la posición táctica. Se realizaron pruebas de comparación múltiple post hoc de Bonferroni para identificar diferencias 
significativas. La significación se estableció al $p<0,05$. Todos los cálculos se realizaron utilizando el software SPSS v.21.0 para Windows.

\section{RESULTADOS}

La Tabla 1 muestra el número de EMAVS, la duración de los mismos, la FC alcanzada y el intervalo de tiempo entre EMAVS.

No se observaron diferencias significativas entre los jugadores de primera y segunda división en relación a las variables antropométricas: altura (180,0 \pm 0 , $07 \mathrm{~cm}$ vs. $180,4 \pm 5.7 \mathrm{~cm}[\mathrm{p}>0.01])$, peso $(75,13 \pm 7.59 \mathrm{~kg}$ vs. $77,4 \pm 5,9 \mathrm{Kg}[\mathrm{p}>$ $0,01])$, o edad $(24,36+/ 2,93$ años vs. $26,2+/-3,76$ años; $p>0,01$.

Número de EMAVS, su duración y el intervalo entre ellos

No se observaron diferencias significativas entre los jugadores de primera y segunda división en la duración de los EVAMS (Figura 1), ni en el intervalo entre ellos (Figura 2), ( $p>0,05$ en ambos casos).

Los delanteros de segunda división mostraron un mayor número de EMAVS en comparación a los de primera división $(p=0,025)$. Para el resto de posiciones no se observaron diferencias significativas en el número de EMAVS entre los jugadores de primera y segunda división ( $p>0,05)$ (Figura 3).

Tabla 1: Número medio de esfuerzos intermitentes de muy alta velocidad de carrera y esprint (EMAVS), duración e intervalo de tiempo entre ellos. Frecuencia cardiaca (FC) durante los EMAVS en primera y segunda división, y entre las diferentes posiciones tácticas.

\section{División Posición N EMAVS Duración Intervalo FC (Ipm) EMAVS entre EMAVS}

(s)

\begin{tabular}{cccccc}
$1^{\text {a }}$ & $D(n=8)$ & $25,36 \pm 6,41$ & $2,59 \pm 0,40$ & $109,41 \pm 28,10$ & $164,40 \pm 10,92$ \\
& $M(n=9)$ & $30,61 \pm 6,91$ & $2,56 \pm 0,38$ & $126,84 \pm 143,02$ & $165,45 \pm 15,43$ \\
& $F(n=4)$ & $31,57 \pm 6,05$ & $2,80 \pm 0,37$ & $85,14 \pm 17,89$ & $163,10 \pm 6,48$ \\
$2^{a}$ & $D(n=6)$ & $25,56 \pm 8,52$ & $2,35 \pm 0,53$ & $105,33 \pm 28,36$ & $174,74 \pm 9,90$ \\
& $M(n=5)$ & $27,35 \pm 9,28$ & $2,57 \pm 0,54$ & $64,75 \pm 8,18$ & $160,66 \pm 14,07$ \\
& $F(n=4)$ & $42,25 \pm 6,85$ & $2,66 \pm 0,28$ & $108,82 \pm 44,98$ & $165,22 \pm 6,41$ \\
Total & $n=36$ & $28,35 \pm 8,35$ & $2,58 \pm 0,44$ & $107,07 \pm 67,00$ & $164,92 \pm 12,39$ \\
& \multicolumn{5}{c}{ D: defensas. M: mediocampistas. F: delanteros. }
\end{tabular}




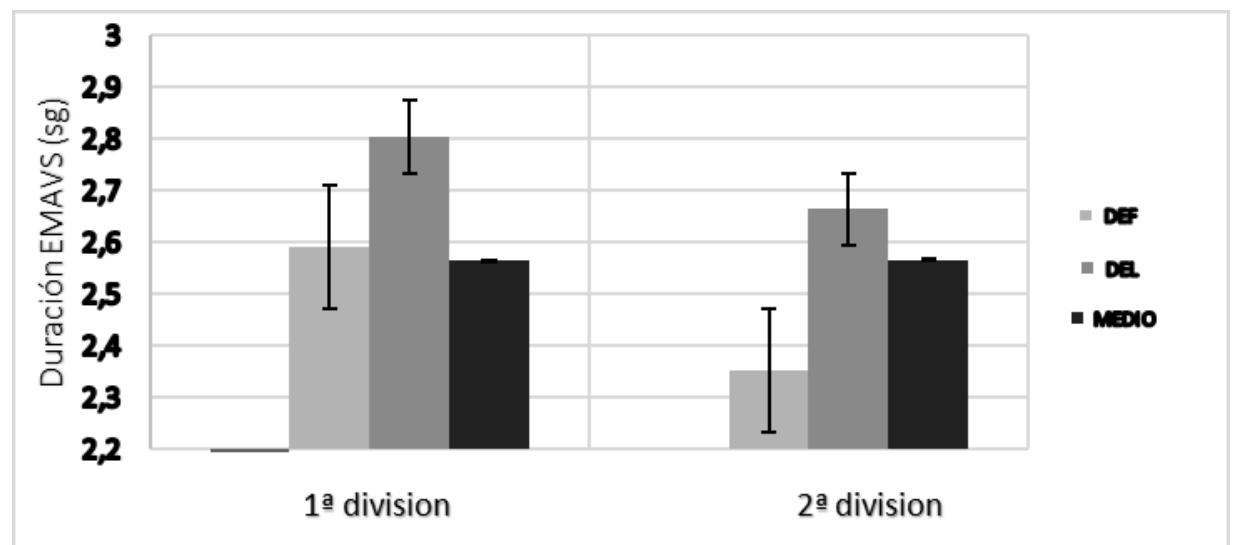

Figura 1: duración de los EMAVS entre jugadores de primera y segunda división, según su posición táctica.

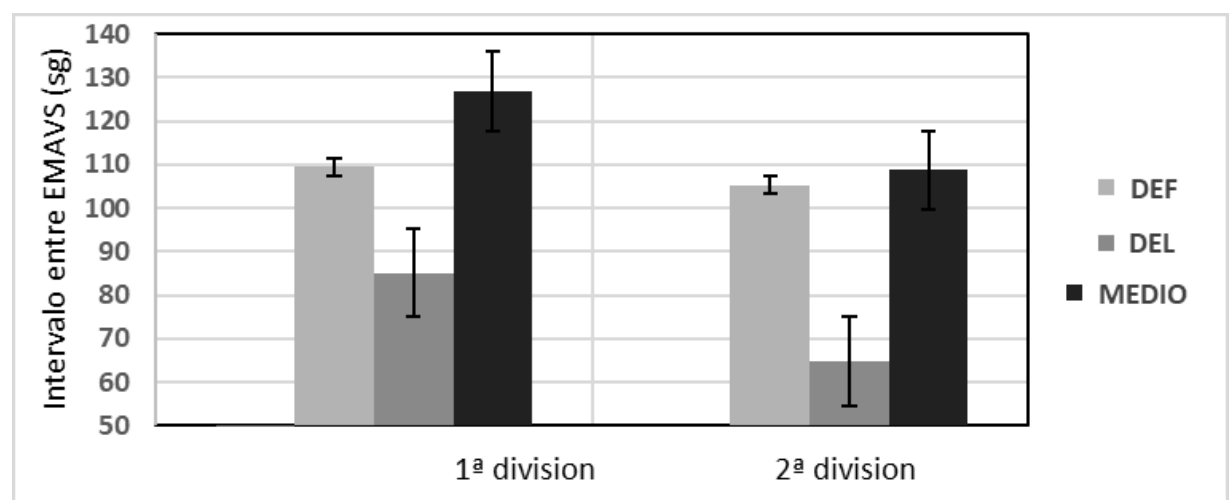

Figura 2. Tiempo de los intervalos entre EMAVS (sg) entre jugadores de primera y segunda división, según su posición táctica.

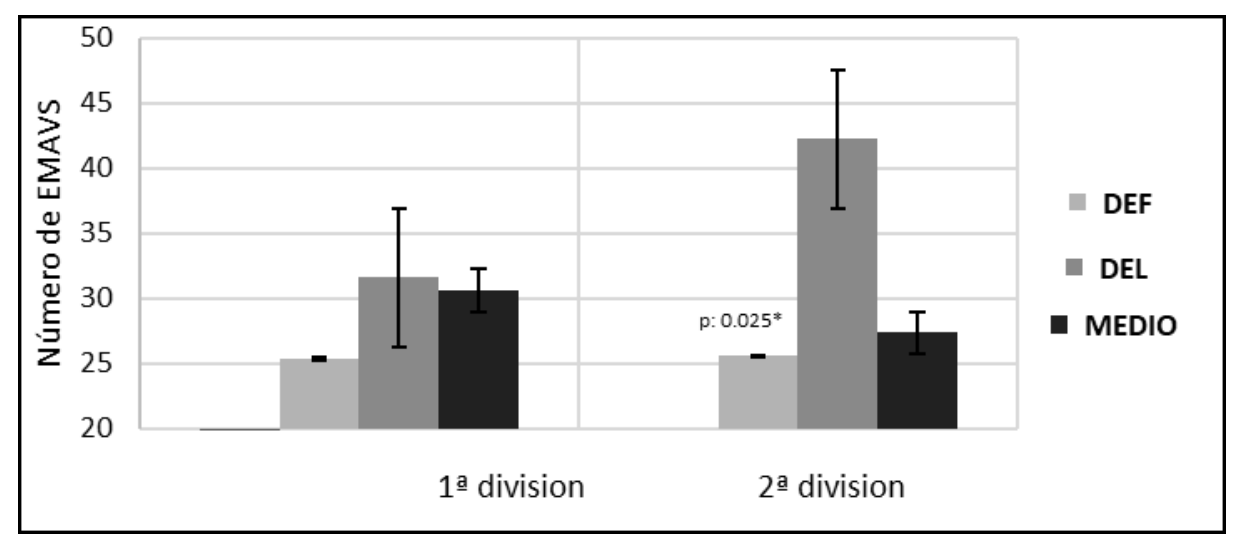

Figura 3. Número de EMAVS entre jugadores de primera y segunda división, según su posición táctica.

${ }^{*}$ Diferencias significativas

\section{Frecuencia cardiaca}

No se observaron diferencias significativas entre los jugadores de la primera y segunda división en la FC media alcanzada durante los EMAVS $(164,50+/-11,7$ vs. $165,50+/-13,44$ latidos por minuto respectivamente $[p=0,45])$, o entre jugadores de diferente posición táctica $(p=0,13)$ (Figura 4). 


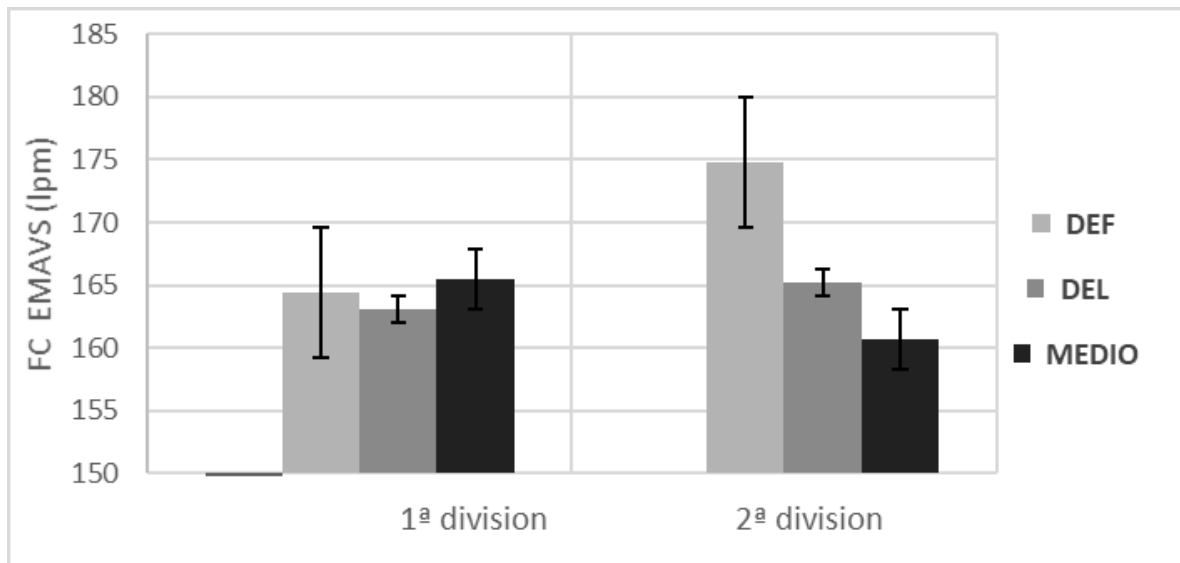

Figura 4. Frecuencia cardiaca media (FC) durante los EMAVS entre jugadores de primera y segunda división, según su posición táctica.

\section{DISCUSIÓN}

El presente artículo, tuvo como objetivo comparar el número, duración y recuperación de los EMAVS durante competición entre futbolistas de primera y segunda división. Como principal hallazgo de nuestro estudio no encontramos diferencias significativas en las variables analizadas entre los jugadores de primera y segunda división. Secundariamente, la descripción de este tipo de esfuerzos y la respuesta fisiológica puede permitir el diseño específico del entrenamiento interválico para la mejora de los EMAVS.

Durante un partido de fútbol, los jugadores realizan una media de $28,35 \pm 8,35$ EVAMS de 2,58 $\pm 0,44 \mathrm{sg}$ de duración y con un intervalo medio entre ellos de $107,07 \pm 67,00 \mathrm{sg}$, alcanzando una FC media de $164,92 \pm 12,39$ p.p.m, sin diferencias significativas entre los jugadores de primera y segunda división, ni entre los jugadores en diferentes posiciones, en términos de la duración de EMAVS, la duración de los intervalos entre los mismos o la FC alcanzada durante estos esfuerzos, tampoco se han encontrado diferencias entre posiciones tácticas a excepción de los delanteros de segunda división. Las variaciones individuales en estos datos y el disponer de dos criterios de evaluación: los tiempos de esfuerzos y las frecuencias cardiacas durante los mismos, permitiría diseñar entrenamientos interválicos individuales, en los que el jugador debería alcanzar durante los esfuerzos de máxima intensidad el $85 \%$ de su FC, con recuperaciones no superiores a $107 \mathrm{sg}$, dichos datos irían variando en el trascurso de la temporada, en función de los datos obtenidos durante los partidos.

El número de EMAVS en los partidos de fútbol masculino y femenino ha sido registrada por otros autores (Bradley et al., 2010; Di Salvo et al., 2009; Gabbett, Wiig, \& Spencer, 2013; Ingebrigtsen, Dalen, Hjelde, Drust, \& Wisløff, 2015). Pero hasta el momento ningún estudio ha asociado la FC a la duración de los esfuerzos, ni a la recuperación entre los mismos, ni han comparado los resultados entre futbolistas profesionales de diferentes categorías. La duración de los EMAVS fueron similares a los reportados por otros autores para otras ligas europeas de primera división (Bradley et al., 2010; Di Salvo et al., 2009; 
Ingebrigtsen et al., 2015) y más altos que las registradas en ligas menos competitivas (Ingebrigtsen et al., 2015). Todos los autores mencionados encontraron diferencias en la duración de los EMAVS entre las diferentes posiciones, con una disminución de los EVAMS en los centrales, a diferencia del presente estudio. Al encontrar diferencias entre posiciones, se podrían establecer grupos por posición en el entrenamiento interválico. No obstante, la medición de datos durante la competición tendría por objeto establecer intensidades individuales de entrenamiento, para lo cual también se podría utilizar la FC.

La FC es una variable comúnmente utilizada en la monitorización de la carga interna de entrenamiento (Alexandre et al., 2012). Algunos autores han informado diferencias en la FC medida durante los partidos de fútbol de los jugadores en diferentes divisiones (Proietti et al., 2017), pero no encontramos ningún estudio que describa el comportamiento de la $\mathrm{FC}$ en los periodos de intervalo entre EMAVS, ni entre jugadores de diferentes posiciones. Las FC registradas alcanzaron aproximadamente el $85 \%$ del máximo teórico, similar a lo informado por otros autores (Bloomfield, Polman, \& O'Donoghue, 2007; Bradley et al., 2009; Dellal et al., 2008; Stolen et al., 2005). Sin embargo, nunca se ha establecido una relación sólida entre la frecuencia cardíaca y el rendimiento (McLaren et al., 2018); por lo tanto, algunos autores recomiendan utilizar diferentes variables para guiar el diseño del entrenamiento interválico (Viana et al., 2018). La medición de los esfuerzos y los tiempos de recuperación entre ellos durante los partidos, aporta información para utilizar en el diseño de este tipo de entrenamientos interválicos.

Actualmente existe una gran controversia en relación a los esfuerzos máximos y supramáximos con respecto al término alta intensidad. En el presente trabajo se han tenido en cuenta aquellos esfuerzos realizados por encima de $19,8 \mathrm{~km} / \mathrm{h}$ (Bishop et al., 2011; Bradley et al., 2010; Rampinini et al., 2007), con el objetivo de englobar todos los esfuerzos de alta intensidad y así describir las respuesta de carga interna expresada a través de la FC.

La FC de los jugadores durante un partido únicamente ha mostrado tener utilidad para monitorizar la respuesta interna del jugador. En entrenamientos específicos para la mejora del rendimiento, es necesario, aportar información tanto de la carga interna (FC) como de parámetros de carga externa (número y duración de EMAVS) con el fin de conocer la exigencia de estos entrenamientos y comparar esos estímulos con los realizados en competición.

En cuanto a las limitaciones del estudio debemos señalar que se trata de un descriptivo donde se analizó un bajo número de partidos. Investigaciones futuras deberían analizar un mayor número de partidos para conocer si existen diferencias entre los jugadores de diferentes categorías y diferentes posiciones. También se debe recordar que los esfuerzos máximos que un jugador puede hacer durante un partido dependen también de su condición física individual (fuerza, resistencia a la fatiga, etc.) en el momento del estudio, así como las condiciones climáticas, las características del campo de juego o la disposición táctica del equipo (Girard et al., 2011). Finalmente, los datos obtenidos fueron 
realizados durante el primer mes de competición al objeto de servir de evaluación inicial. Sería recomendable seguir evaluando dichos parámetros en diversos momentos de la temporada.

\section{CONCLUSIONES}

No se encontraron diferencias significativas en el número, duración de los EMAVS, los intervalos entre ellos y la FC media alcanzada durante los mismos entre los jugadores de fútbol de primera y segunda división, o entre jugadores de diferentes posiciones tácticas. Solo los delanteros realizaron mayor número de EMAVS. Esta información puede ser de gran utilidad para la planificación de los entrenamientos interválicos (duración y recuperación de esfuerzos) que persiguen la mejora de esta variable.

\section{REFERENCIAS BIBLIOGRÁFICAS}

Alexandre, D., da Silva, C. D., Hill-Haas, S., Wong del, P., Natali, A. J., De Lima, J. R., ... Karim, C. (2012). Heart rate monitoring in soccer: interest and limits during competitive match play and training, practical application. J Strength Cond Res, 26(10), 2890-2906. doi:10.1519/JSC.0b013e3182429ac7

Andersson, H. A., Randers, M. B., Heiner-Moller, A., Krustrup, P., \& Mohr, M. (2010). Elite female soccer players perform more high-intensity running when playing in international games compared with domestic league games. J Strength Cond Res, 24(4), 912-919. doi:10.1519/JSC.0b013e3181d09f21

Bishop, D., \& Claudius, B. (2005). Effects of induced metabolic alkalosis on prolonged intermittent-sprint performance. Med Sci Sports Exerc, 37(5), 759-767.

Bishop, D., Edge, J., Davis, C., \& Goodman, C. (2004). Induced metabolic alkalosis affects muscle metabolism and repeated-sprint ability. Med Sci Sports Exerc, 36(5), 807-813.

Bishop, D., Girard, O., \& Mendez-Villanueva, A. (2011). Repeated-sprint ability part II: recommendations for training. Sports Med, 41(9), 741-756. doi:10.2165/11590560-000000000-00000

Bloomfield, J., Polman, R., \& O'Donoghue, P. (2007). Physical Demands of Different Positions in FA Premier League Soccer. J Sports Sci Med, 6(1), 63-70.

Bradley, P. S., Di Mascio, M., Peart, D., Olsen, P., \& Sheldon, B. (2010). Highintensity activity profiles of elite soccer players at different performance levels. J Strength Cond Res, 24(9), 2343-2351. doi:10.1519/JSC.0b013e3181aeb1b3

Bradley, P. S., Sheldon, W., Wooster, B., Olsen, P., Boanas, P., \& Krustrup, P. (2009). High-intensity running in English FA Premier League soccer matches. J Sports Sci, 27(2), 159-168. doi:10.1080/02640410802512775

Buchheit, M., Mendez-villanueva, A., Simpson, B. M., \& Bourdon, P. C. (2010). Repeated-sprint sequences during youth soccer matches. Int J Sports Med, 31(10), 709-716. doi:10.1055/s-0030-1261897 
Buchheit, M., Simpson, B. M., \& Mendez-Villanueva, A. (2013). Repeated highspeed activities during youth soccer games in relation to changes in maximal sprinting and aerobic speeds. Int J Sports Med, 34(1), 40-48. doi:10.1055/s-0032-1316363

Dellal, A., Chamari, K., Pintus, A., Girard, O., Cotte, T., \& Keller, D. (2008). Heart rate responses during small-sided games and short intermittent running training in elite soccer players: a comparative study. J Strength Cond Res, 22(5), 1449-1457. doi:10.1519/JSC.0b013e31817398c6

Di Salvo, V., Gregson, W., Atkinson, G., Tordoff, P., \& Drust, B. (2009). Analysis of high intensity activity in Premier League soccer. Int $J$ Sports Med, 30(3), 205-212. doi:10.1055/s-0028-1105950 [doi]

Duffield, R., King, M., \& Skein, M. (2009). Recovery of voluntary and evoked muscle performance following intermittent-sprint exercise in the heat. Int J Sports Physiol Perform, 4(2), 254-268.

Gabbett, T. J., \& Mulvey, M. J. (2008). Time-motion analysis of small-sided training games and competition in elite women soccer players. $J$ Strength Cond Res, 22(2), 543-552. doi:10.1519/JSC.0b013e3181635597

Gabbett, T. J., Wiig, H., \& Spencer, M. (2013). Repeated high-intensity running and sprinting in elite women's soccer competition. Int J Sports Physiol Perform, 8(2), 130-138.

Gantois, P., Aidar, F. J., Gama de Matos, D., De Souza, R. F., Da Silva, L. M., De Castro, K. R., . . . Cabral, B. (2017). Repeated sprints and the relationship with anaerobic and aerobic fitness of basketball athletes. Journal of Physical Education and Sport., 17(2), 910-915.

Girard, O., Mendez-Villanueva, A., \& Bishop, D. (2011). Repeated-sprint ability part I: factors contributing to fatigue. Sports Med, 41(8), 673-694. doi:10.2165/11590550-000000000-00000

Haseler, L. J., Hogan, M. C., \& Richardson, R. S. (1999). Skeletal muscle phosphocreatine recovery in exercise-trained humans is dependent on O2 availability. J Appl Physiol (1985), 86(6), 2013-2018. doi:10.1152/jappl.1999.86.6.2013

Impellizzeri, F. M., Rampinini, E., Castagna, C., Bishop, D., Ferrari Bravo, D., Tibaudi, A., \& Wisloff, U. (2008). Validity of a repeated-sprint test for football. Int J Sports Med, 29(11), 899-905. doi:10.1055/s-2008-1038491 [doi]

Ingebrigtsen, J., Dalen, T., Hjelde, G. H., Drust, B., \& Wisløff, U. (2015). Acceleration and sprint profiles of a professional elite football team in match play. European journal of sport science, 15(2), 101-110.

McLaren, S. J., Macpherson, T. W., Coutts, A. J., Hurst, C., Spears, I. R., \& Weston, M. (2018). The Relationships Between Internal and External Measures of Training Load and Intensity in Team Sports: A MetaAnalysis. Sports Med, 48(3), 641-658. doi:10.1007/s40279-017-0830-z

Proietti, R., di Fronso, S., Pereira, L. A., Bortoli, L., Robazza, C., Nakamura, F. Y., \& Bertollo, M. (2017). Heart Rate Variability Discriminates Competitive Levels in Professional Soccer Players. J Strength Cond Res, 31(6), 1719-1725. doi:10.1519/JSC.0000000000001795

Rampinini, E., Bishop, D., Marcora, S. M., Ferrari Bravo, D., Sassi, R., \& Impellizzeri, F. M. (2007). Validity of simple field tests as indicators of 
match-related physical performance in top-level professional soccer players. Int J Sports Med, 28(3), 228-235. doi:10.1055/s-2006-924340

Rodriguez-Fernandez, A., Sanchez Sanchez, J., Rodriguez-Marroyo, J. A., Casamichana, D., \& Villa, J. G. (2017). Effects of 5-week pre-season small-sided-game-based training on repeat sprint ability. J Sports Med Phys Fitness, 57(5), 529-536. doi:10.23736/S0022-4707.16.06263-0

Stolen, T., Chamari, K., Castagna, C., \& Wisloff, U. (2005). Physiology of soccer: an update. Sports Med, 35(6), 501-536.

Taylor, J., Macpherson, T., Spears, I., \& Weston, M. (2015). The effects of repeated-sprint training on field-based fitness measures: a meta-analysis of controlled and non-controlled trials. Sports Med, 45(6), 881-891. doi:10.1007/s40279-015-0324-9

Viana, R. B., de Lira, C. A. B., Naves, J. P. A., Coswig, V. S., Del Vecchio, F. B., Ramirez-Campillo, R., . . Gentil, P. (2018). Can We Draw General Conclusions from Interval Training Studies? Sports Med. doi:10.1007/s40279-018-0925-1

Wragg, C. B., Maxwell, N. S., \& Doust, J. H. (2000). Evaluation of the reliability and validity of a soccer-specific field test of repeated sprint ability. Eur $J$ Appl Physiol, 83(1), 77-83. doi:10.1007/s004210000246

Número de citas totales/ Total references: 28 (100\%)

Número de citas propias de la revista/ Journal's own references: $0(0 \%)$

Rev.int.med.cienc.act.fís.deporte - vol. 21 - número 81 - ISSN: 1577-0354 